\title{
Simulation of plasma processes for microelectronic fabrication*
}

\author{
Ralf Peter Brinkman ${ }^{1} \uparrow$, Matthias Kratzer ${ }^{2}$ and Harald Schmidt ${ }^{3}$ \\ ${ }^{1}$ Infineon AG, Otto-Hahn-Ring 6, 81370, München, Germany; ${ }^{2}$ Institut für Technische \\ Elektrophysik, TU München, Germany; ${ }^{3}$ Lehrstuhl für Numerische Mathematik, \\ LMU München, Germany
}

\begin{abstract}
An approach is presented which allows to predict important characteristics of plasma based surface modification techniques like reactive ion etching (RIE), plasma etching (PE), ionized metal vapor deposition (IPVD), or plasma enhanced physical vapor deposition (PECVD). In a first step, the electrical field in the vicinity of the substrate is calculated by means of a self-consistent plasma boundary sheath model. In a second step, this field is used to calculate the energy and angular distribution of the ions impinging the surface. The knowledge of this distribution allows a more realistic prediction of essential process properties like the maximum aspect ratio of an etch process, or the obtainable conformality of a deposition step.
\end{abstract}

\section{INTRODUCTION}

Radio frequency (RF) driven low pressure gas discharges or 'plasmas' form the basis of many important process steps in microelectronics fabrication and other advanced industries. Notable examples are plasma etching (PE), reactive ion etching (RIE), plasma enhanced physical vapor deposition (PECVD), ionized metal vapor deposition (IPVD), and resist ashing.

The performance of plasma based manufacturing processes depends on the properties of the driving discharge in two different ways. First, the obtainable etch and deposition rates depend on the flux of the energetic particles-ions and activated neutrals—which reach the substrate. These particles are generated by electron-neutral interactions which take place throughout the plasma volume; the flux rate is thus dependent mainly on the properties of the plasma bulk. In a second, more critical way, however, it is the dynamics of the immediate vicinity of the substrate which influences the process characteristics: The strong electric field present in the boundary sheath, together with the collisional interaction with the neutral background gas, determine the energy and angular distribution of the positive ions impinging upon the surface. This distribution, in turn, determines most of the qualitative features of the plasma process. In the case of VLSI manufacturing, these are properties like the maximum aspect ratio of an etch process, or the obtainable conformality of a deposition step. Detrimental effects which occur in the processing steps, like plasma damage, can also be described in these terms.

In order to understand and predict the properties of plasma based manufacturing processes, it is therefore mandatory to understand the distribution function of the incoming energetic particles. The calculation of the absolute flux densities, on the other hand, is dispensable as long as one is willing to adjust for an overall scale factor. For this reason, we concentrate in this work on the dynamics of the boundary sheath, and evaluate a detailed model of the interaction between the charges, the neutral particles and the fields in this region. This model, called the hybrid boundary sheath model, will be outlined in the next section.

It is obvious that a calculation of the properties of the substrate-incident particles is only the first step

*Lecture presented at the 14th International Symposium on Plasma Chemistry, Prague, Czech Republic, 2-6 August 1999, pp. 1809-1918.

$\dagger$ Corresponding author. 
toward a complete description of a given plasma process. Equally important is to understand in detail how the impinging articles interact with the substrate surface and modify its properties: What exactly happens if a certain particle with a given energy hits a substrate at a particular angle? Recently, much effort has been devoted to study this question from a molecular dynamics point of view (e.g. [1]); a discussion of this subject, however, lies beyond the scope of this contribution.

When both sets of information, the knowledge of particle distribution and the knowledge of the surface reactivity, are available, one can predict the temporal evolution of the substrate characteristics under the given conditions. In microelectronics, this study is referred to as feature scale or topography simulation.

In summary, one can state that a comprehensive simulation of plasma surface modification techniques requires a hierarchy of models which cover all relevant scales of the dynamics from the reactor scale $(\approx 10 \mathrm{~cm})$ down to the scale of the atomar surface processes $(\approx 0.1 \mathrm{~nm})$. Figure 1 depicts a schematic representation of this idea. It is clear that the predictive prowess of the overall simulation depends on the reliability of all the sub-models invoked: much work in this direction is still to be done. We will, however, illustrate the approach in the last section by presenting the results of a first application. The process to be studied will be the filling of VLSI contact holes using the process of ionized metal vapor deposition.

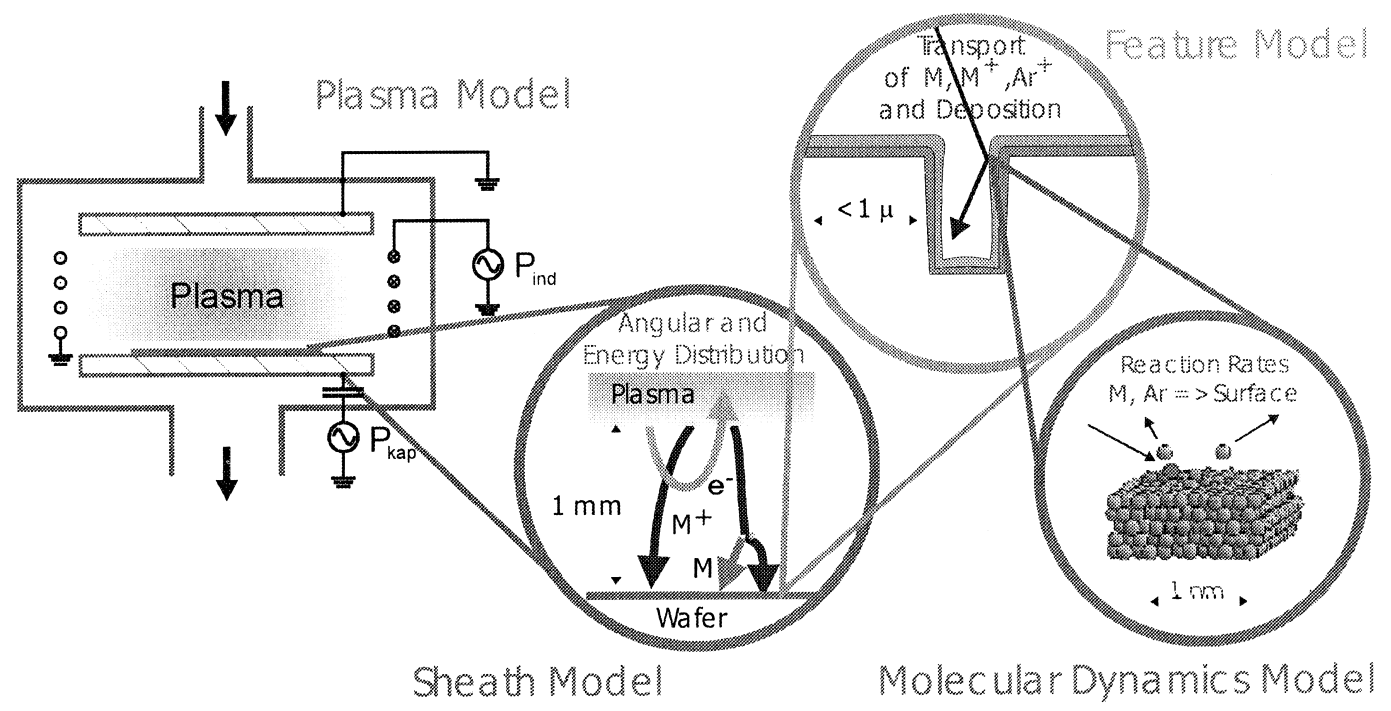

Fig. 1 Schematic representation of the model hierarchy required for predictive plasma process simulation.

\section{THE HYBRID BOUNDARY SHEATH MODEL}

Our approach to calculate the energy and angular distribution of the energetic particles which strike the surface consists of two steps. In the first step, we calculate the electrical field above the substrate as a function of position and time. For this purpose we employ a self-consistent boundary sheath model which is based on a fluid-dynamic description of the discharge plasma. In the second step, we follow the paths of a sufficient number of ions through this field until they reach the surface, taking into account collisions with the neutral background by means of a Monte Carlo scattering method. Together, the two modules form the hybrid boundary sheath model as depicted in Fig. 2.

The fluid-dynamic sheath model solves the macroscopic equations of motion of the plasma (which consists of electrons and an arbitrary number of positive ion species) simultaneously with Poisson's equation. Negative ions could also be incorporated into the approach, but they are strongly repelled by the sheath field and we will neglect their presence in the following. The model derivation adopts a one-dimensional approximation, using the rationale that the sheath thickness is much smaller than any geometry scale, and assumes that the RF modulation (if present) lies in the intermediate range between the ion and the electron plasma frequencies, $\omega_{\mathrm{pi}} \ll \omega_{\mathrm{RF}} \ll \omega_{\mathrm{pe}}$. Furthermore, periodic behavior with respect to the RF period is postulated, or stationarity in the case of a non-modulated DC sheath. 


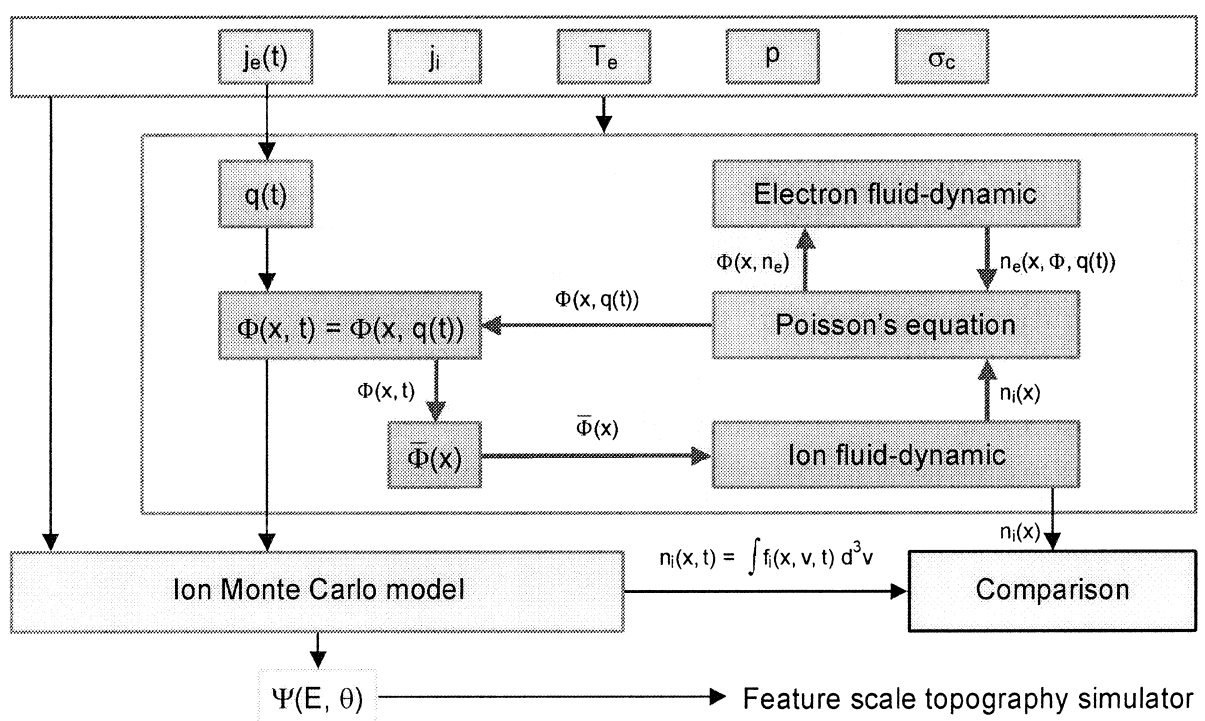

Fig. 2 Schematic representation of the hybrid boundary sheath module.

Under these assumptions, repeated application of the method of asymptotic time and length scale expansion allows to reduce the original description of the sheath dynamics - which consists of a system of coupled partial differential equations in $x$ and $t$-to a system of ordinary differential equations in a transformed spatial coordinate $q$. The dependent variables of this system of equations are the ion densities $\mathrm{n}_{\alpha}$, where the index runs over all positive ion species. A remarkable feature of the model is that is does not require boundary conditions, the degrees of freedom of the differential equations are removed by internal consistency requirements. A more detailed description of the sheath model will be presented elsewhere [2].

From the solution of the sheath equation, which self-consistently gives the spatial distribution of the ions in the sheath and the average values of the electron density and the field strength, we can deduct also all other relevant information, notably the time-resolved values of the electrical potential and the electrical field.

It is the latter quantity which enters the second module of the hybrid model, a one-particle Monte Carlo code which follows the path of the incoming positive ions (and possibly also that of the generated energetic neutrals), until they strike the substrate surface or are scattered back into the plasma bulk. Collisional interaction with the neutral background (charge exchange, elastic collisions-both hard sphere and Langevin enhanced) is introduced effectively via a null collision method. (Multi-body or Coulomb interactions are not considered due to their relative unimportance.) Averaging over a sufficiently high number of ion trajectories yields the desired distribution functions; to arrive at absolute values, the number of monitored ions of each species is adjusted corresponding to the respective flux.

Note that the fluid-based sheath module is self-consistent, but the overall hybrid model is not: The information calculated in the Monte Carlo module, notably the particle density, is not fed back into the field model. Instead, we use the comparison between the ion densities of the fluid model and those of the kinetic model to estimate the model error related to the simplifications implicit in the fluid model.

A typical outcome of this comparison is depicted in Fig. 3, which shows the fluid dynamic and the Monte Carlo density in the sheath of a high density argon plasma under RF modulation. From the excellent agreement we infer that neither of the two simplifying assumptions which are used to calculate the electric field - the adoption of a fluid dynamic ion description and the assumption of a stationary ion density-seems to have detrimental effects on the results. In other words, the drastic reduction in computational effort, compared to that of a full PIC calculation, is not paid for by an excessive model 


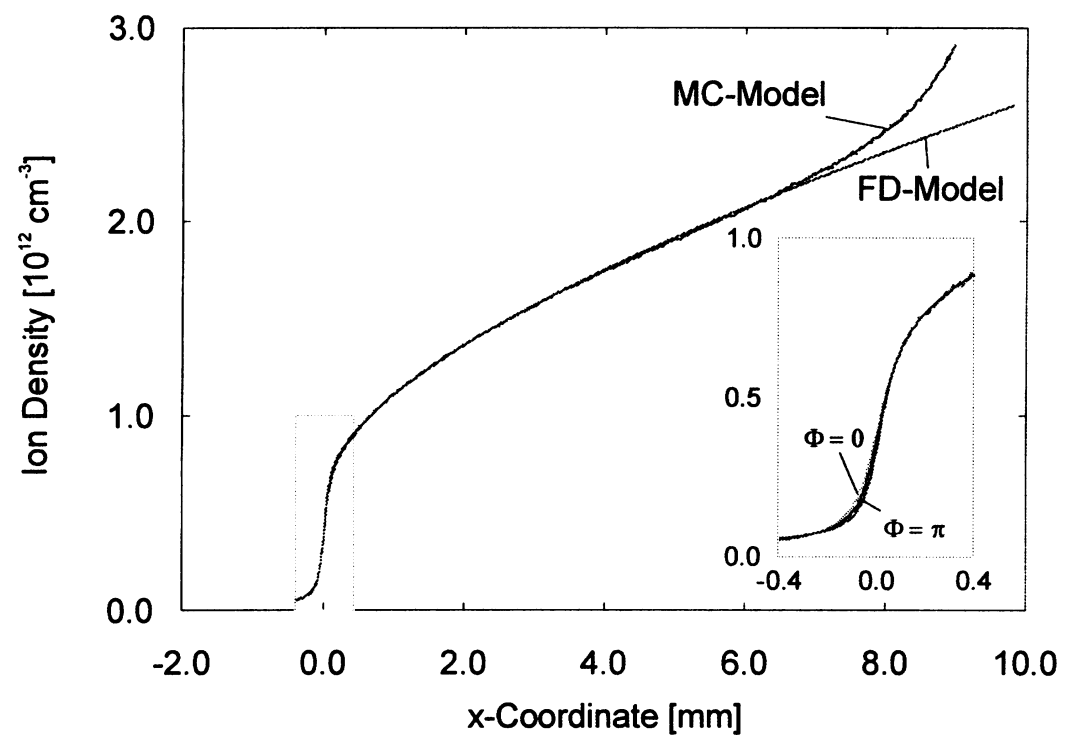

Fig. 3 The ion density in an argon plasma, FD model vs. MC model.

error. (The deviation at $x>8 \mathrm{~mm}$ is due to the fact that the ions, started at $10 \mathrm{~mm}$, have not yet reached equilibrium with the field.)

In addition, a comparison with experimental data yields an excellent agreement. In the following two figures we depict the distribution functions behind an RF sheath, with the discharge gas being $2.4 \mathrm{mTorr}$ argon in Fig. 4, and 3 mTorr oxygen in Fig. 5. The experimental date (curves b) were taken from Kuypers et al. [3]; the calculated distribution functions (curves a) were folded with a Gaussian of $3 \mathrm{eV}$ standard deviation, in order to represent the limited energy resolution of the mass spectrometer.

Based on the consistency check and the agreement with the experimental data, we are positive that our approach gives a faithful representation of sheath dynamics. We now turn to results that cannot be verified that easily, namely to the angular distribution of the incident particles. Figure 6 shows the ion energy

(a)

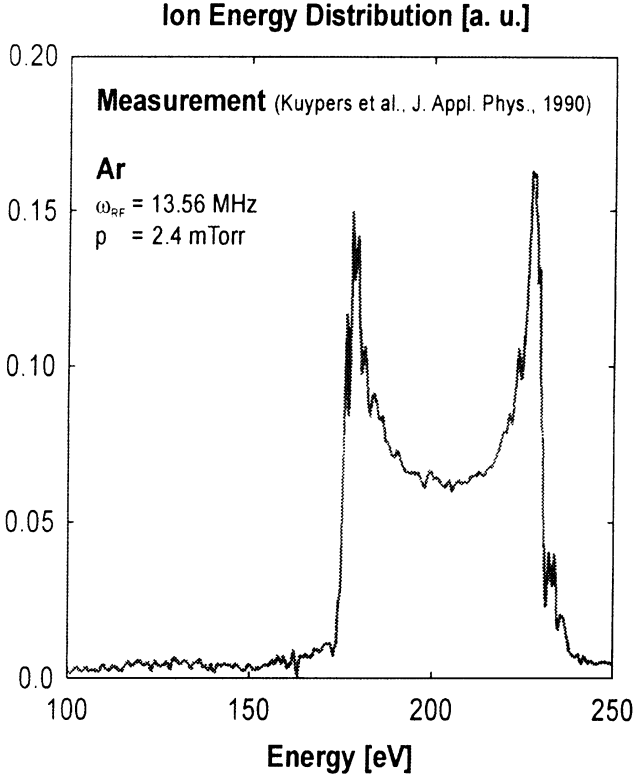

(b)

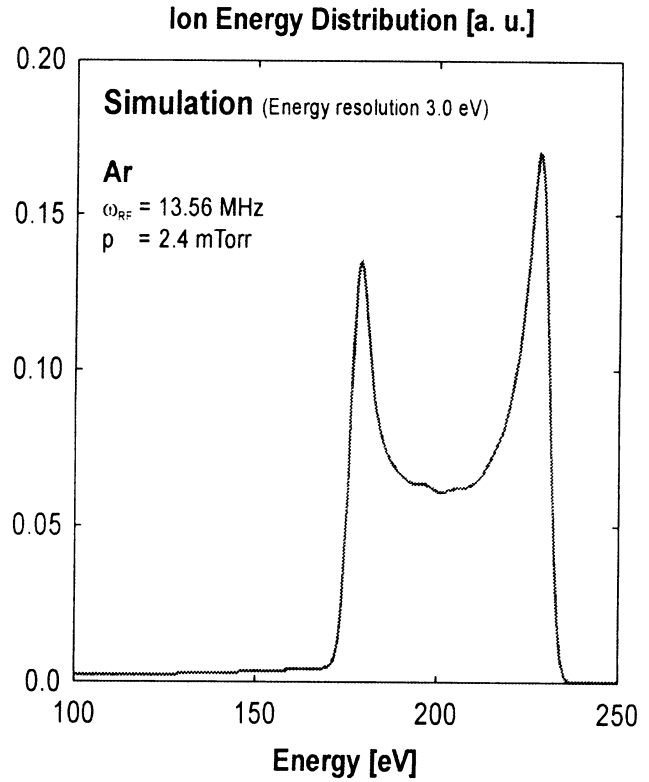

Fig. 4 IEDF behind an oxygen sheath: (a) experimental data of Kuypers et al.; (b) corresponding simulation. 
(a)

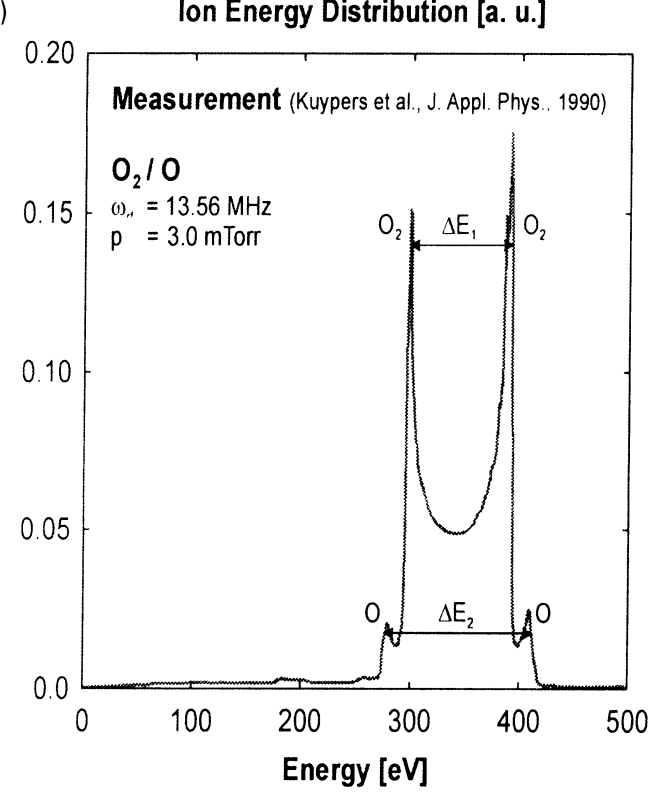

(b)

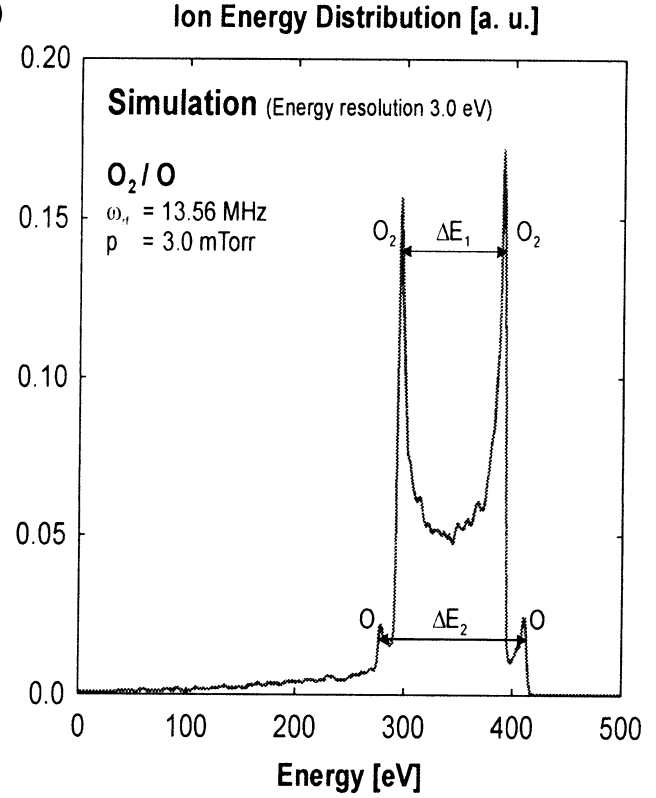

Fig. 5 IEDF behind an oxygen sheath: (a) experimental data of Kuypers et al.; (b) corresponding simulation.

distribution behind the sheath of an $200 \mathrm{mTorr}$ argon plasma subjected a DC bias of $230 \mathrm{~V}$, and the corresponding angular distribution at selected energies.

It can be seen that the angular distribution behind a sheath can have quite a complex structure. In this case, it is essentially the sum of two distinct populations, the narrow one representing those ions that had no collision or a charge exchange collision as their last scattering event, and the broad one representing ions that were elastically scattered in the recent past.

\section{APPLICATION EXAMPLE: CONTACT HOLE FILLING WITH IPVD}

Having outlined how the distribution function of the wafer-incident ions (and possibly that of energetic neutrals) can be calculated, we proceed to describe a typical application of the results, namely the subsequent use of a topography simulator to determine the profile evolution of a microelectronic feature. As an example, we choose the filling of an axi-symmetric contact hole. This research is ongoing, and will be described in more detail elsewhere [4]. Our description here is therefore only a short outline, and shall be taken as strictly preliminary.

The process we will focus on is a special technique to deposit thin metal films on a surface, known as ionized metal vapor deposition or IPVD. It is similar to standard sputtering, in that it utilizes the flux of energetic ions from a low pressure argon plasma into a negatively biased metal substrate to generate a vapor of neutral metal atoms. As opposed to standard sputtering, however, the plasma also serves to ionize a sizeable fraction of this vapor. The generated ions, subject to the electrical field of the plasma, are accelerated towards the material surface and reach it with a forward directed angular distribution. The anisotropic nature of the flux allows for a non-conformal deposition characteristic; IPVD is therefore utilized to fill contact holes and other high aspect ratio structures which would be otherwise prone to void formation.

It is clear that the growth rate of the metal film at a given position of the contact hole will be proportional to the local projected flux, but will depend also on the values of the local sticking coefficient and the resputtering factor. The flux depends on the distribution of the incident particles as well as on the orientation of the surface, and can be influenced by reflection and/or geometric shadowing effects. The sticking and the resputtering coefficients are also functionals of the distribution: the probabilities of a particle to either attach itself to a surface, to get reflected or to induce a sputtering event are strongly dependent on its energy and orientation. 

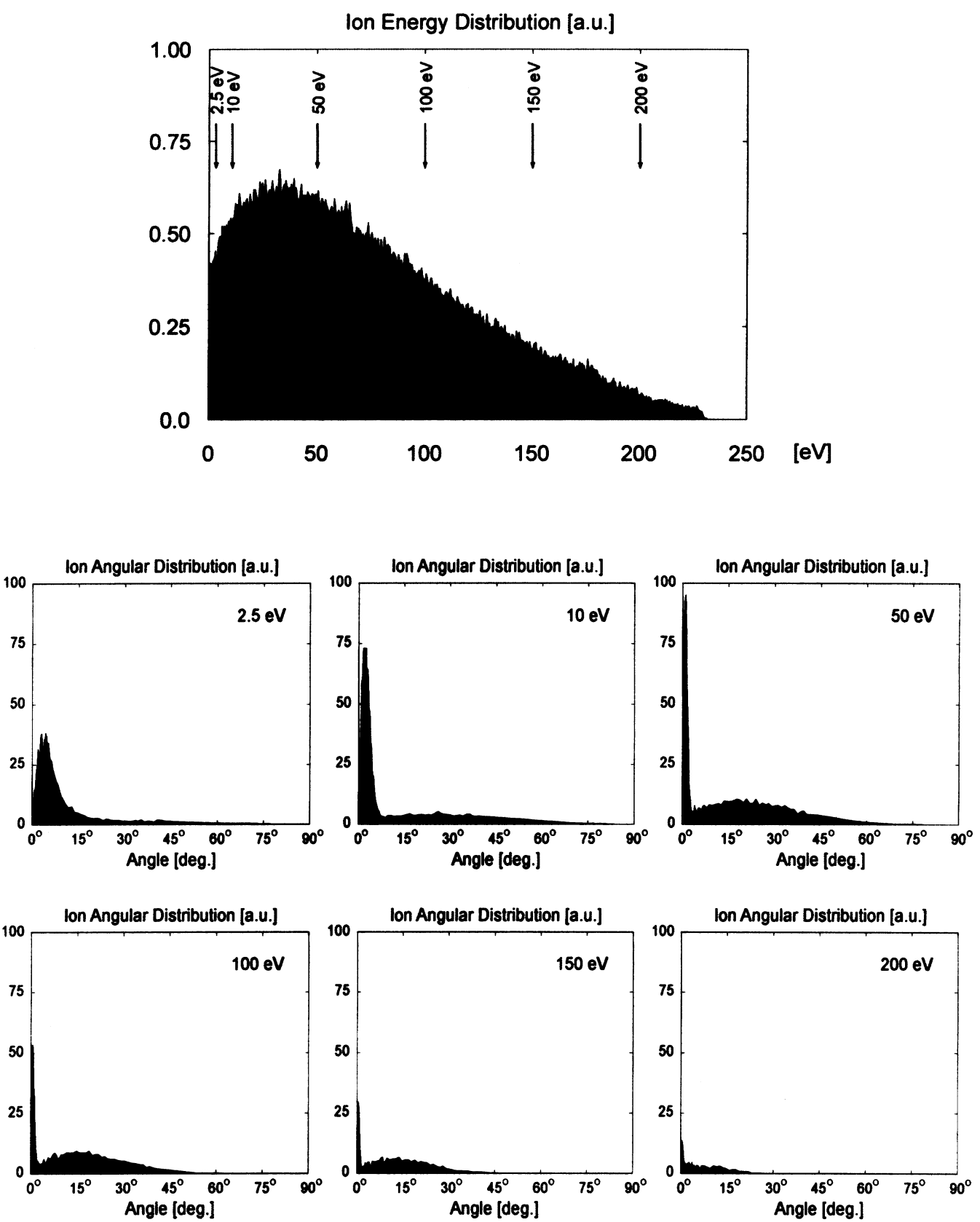

Fig. 6 Ion energy (a) and angular (b) distribution behind an argon sheath (200 mTorr, $230 \mathrm{~V}$ DC bias).

Figure 7 shows the probability of the important elementary events, namely particle absorption, particle reflection, and sputtering, as a function of the energy and the off-normal angle of the incoming ion or neutral. The results have been obtained by a statistical evaluation of a large number of molecular dynamics simulation runs of the interaction of the particle and the surface, details can be found in ref. [5].

Once the effective growth rate of the metal film is known for all locations, the evolution of the contact hole topography can be followed using a feature scale simulation. The following pictures show the results of such a simulation as compared to a corresponding REM image (Fig. 8).

Our experience indicates that the predicted characteristics of the deposited metal film strongly depend on the details of the distribution function of the incident particles. The good quantitative 

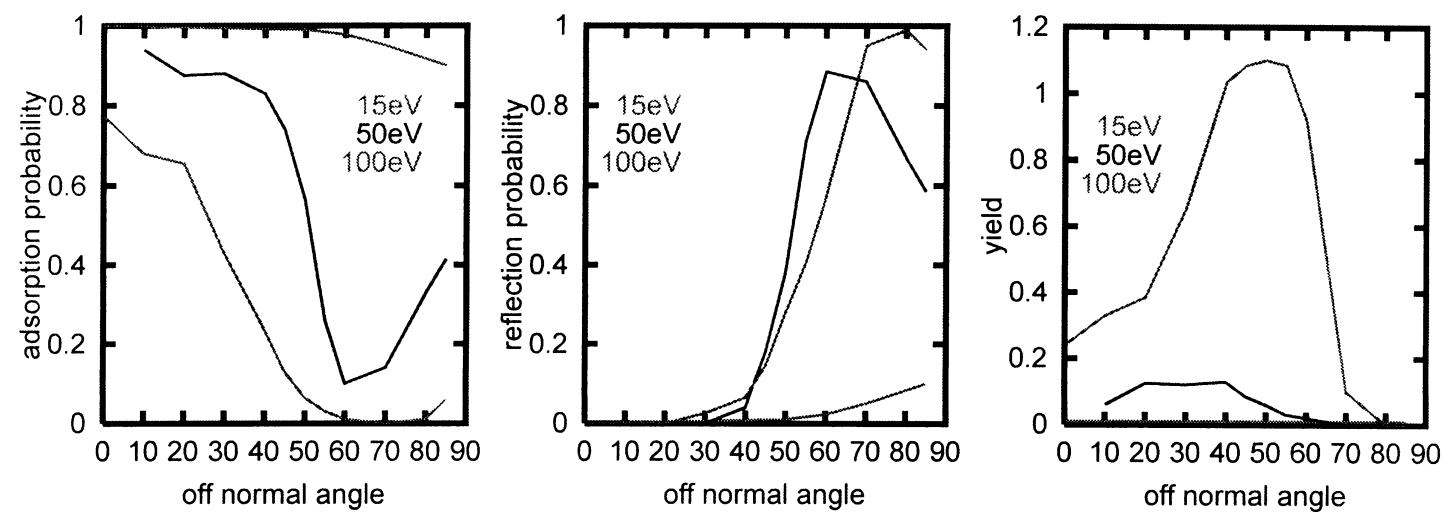

Fig. 7 The probability of adsorption, reflection and sputtering as a function of particle angle and energy [5].

agreement of simulation and experiment can thus be taken as another indication that the distribution function as calculated by our method provides a good approximation to reality. While we would like to stress once more that the results presented in this section are preliminary and are intended mainly as an illustration, we are safe to conclude that the detailed knowledge of the distribution function of the incident particles is indispensable for the predictive simulation of the feature profiles obtained in any IPVD-process.
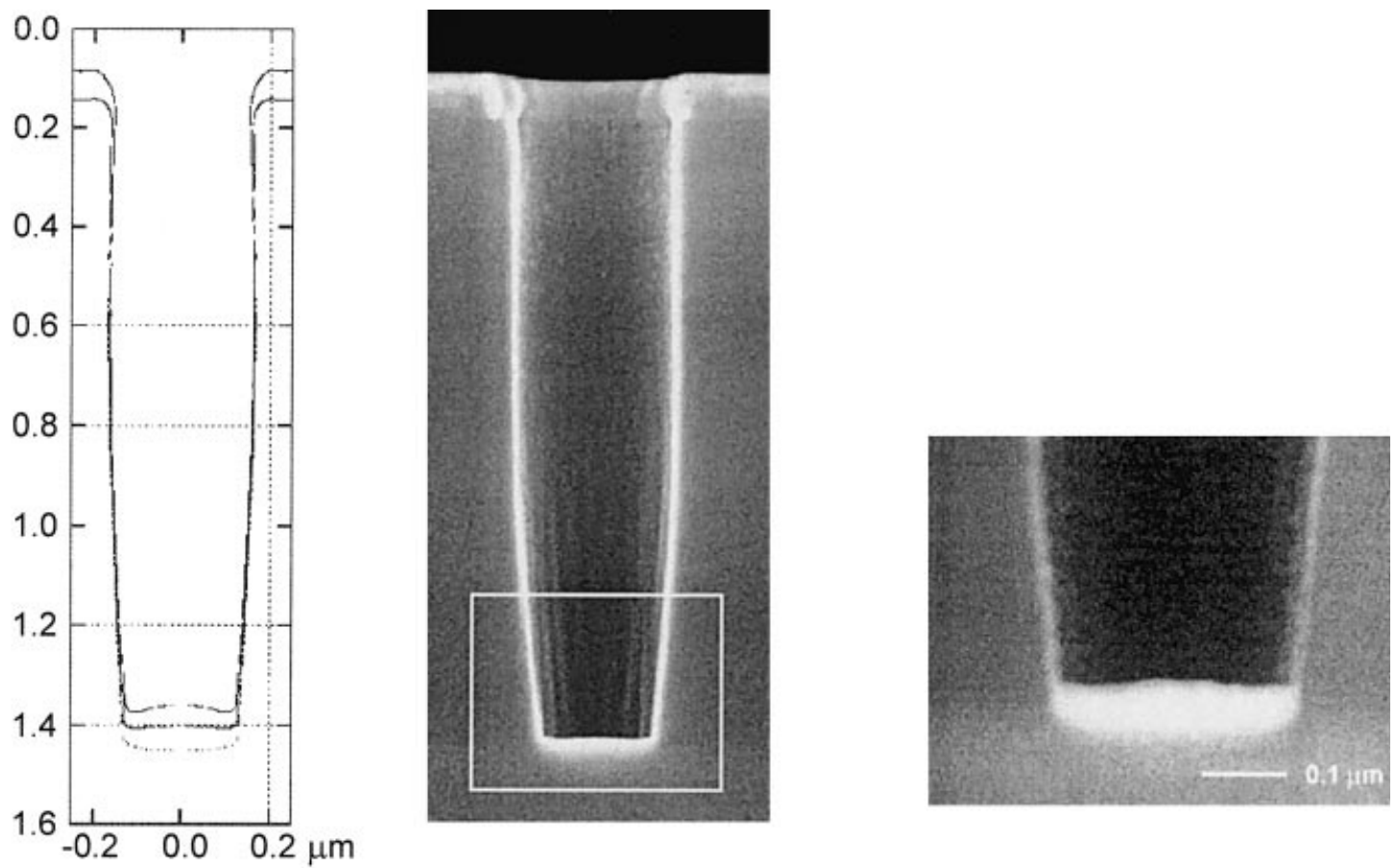

Fig. 8 The metal film deposited in an axi-symmetric contact hole, simulation vs. REM [5].

\section{REFERENCES}

1 U. Hansen, P. Vogl, V. Fiorentini. Phys. Rev. B. 59(12), 7856-7859 (1999).

2 M. Kratzer, R. P. Brinkmann, H. Schmidt. Submitted to J. Appl. Phys.

3 A. D. Kuypers, H. J. Hopman. J. Appl. Phys. 67(3), 1229 (1990).

4 U. Hansen, S. Rodgers, K. F. Jensen. Submitted to Phys. Rev. B.

5 U. Hansen, A. Kersch. Phys. Rev. B 60(20), 14417-14421 (1999). 